\title{
Trust among siblings is intuitive
}

\author{
Victor KM Shiramizu, Iris J Holzleitner, Kieran J O'Shea, Vanessa Fassolt, \\ Lisa M DeBruine
}

Institute of Neuroscience \& Psychology, University of Glasgow, Scotland, UK

Victor.Shiramizu@glasgow.ac.uk

\begin{abstract}
Research on whether prosocial behavior is deliberate or intuitive typically uses decision time in economic games as a proxy for the automaticity of underlying cognitive processes. We investigate the relationship between trust and decision time in a sample with a predicted high baseline level of cooperative behavior and low conflict between self-interested and cooperative motives: biological siblings. Sixty-three full sibling pairs played for money in a one-shot trust game. We found a significant negative linear (rather than quadratic) effect of trust on decision time: the more money a sibling was trusted with, the quicker the decision was made. These results suggest that trust among siblings is intuitive and largely based on automatic and effortless processes.
\end{abstract}

Keywords: trust; kinship; decision time; intuition; prosociality.

\section{Introduction}

The cognitive basis of prosocial behavior can reveal whether cooperative decisions exhibit features of controlled or automatic actions (see review by Zaki \& Mitchell, 2013). Previous studies of this issue have applied the dualprocess perspective of decision-making, which proposes that faster decisions are effortless and intuitive, while slower decisions are more deliberate and effortful (Kahneman, 2011). This topic has been systematically explored through the Social Heuristics Hypothesis, showing that intuition typically favors cooperation (Rand et al., 2014; Rand 2019). Consequently, decision time is often used as a proxy for the cognitive mechanisms underlying cooperation (Evans \& Rand, 2019).

Findings for the relationship between unconstrained (i.e., self-paced) decision time and prosociality are mixed (Spiliopoulos \& Ortmann, 2018). Some studies found negative relationships between cooperation and self-paced decision time during the public goods game, where cooperative decisions were made faster than selfish ones, suggesting that cooperation is intuitive, rather than deliberate (Lotito et al., 2013; Rand et al., 2012). Faster decisions are also related to higher offers during the dictator game and rejections of unfair offers during the ultimatum game (Cappelen et al., 2016; Halali et al., 2011). However, other studies have also reported positive relationships between prosociality and self-paced decision time during social dilemmas 
(Lohse et al., 2017; Piovesan \& Wengström, 2009). Moreover, Evans et al. (2015) found that both selfish and cooperative decisions were faster during prisoner's dilemma and repeated public goods game, suggesting that the relationship between decision time and cooperation is quadratic (inverted- $U$ pattern) rather than linear when the conflict level between self-interested and cooperative motives is low. In other words, if individuals have a clear preference to cooperate or to defect (low conflict level), they make faster decisions than when preference to cooperate or defect is weak or ambivalent (high conflict level).

It has also been noted that studies on humans have focused on the cognitive mechanisms underlying cooperation in non-kin (Rand \& Nowak, 2013). Hamilton's theory of inclusive fitness (1964) demonstrates how altruistic cooperation can be selected among relatives: $r>c / b$, where the probability of sharing a gene $(r)$ must exceed the cost-benefit ratio $(c / b)$ of the altruistic behavior. Moreover, conflict between self-interested and cooperative motives among relatives (e.g. full siblings) tends to decrease once genetic relatedness increases (Schlomer et al., 2011). Indirect evidence using an Implicit Association Test (IAT) found attitude similarity to a target person was automatically associated with kinship cognitions and this implicit similarity-kin association predicted willingness to help similar others, suggesting that similarity attitudes work as heuristic kinship cue (Park \& Schaller, 2005). Additionally, individuals express high trust levels during economic games toward kin (Vollan, 2011) or partners who resemble themselves (DeBruine, 2002) and also report high behavioral and attitudinal altruism toward siblings (Lieberman et al., 2007). Therefore, siblings are predicted to express high baseline prosociality, giving us an opportunity to explore the cognitive underpinnings of prosocial behavior when family members are interacting during social dilemmas.

Taking these observations into account, in the present study we investigated the relationship between prosociality and self-paced decision time in full siblings. Specifically, in this exploratory study, we tested whether a linear or quadratic effect better explains the association between trust and self-paced decision time when siblings were interacting during the one-shot trust game.

\section{Methods}

\section{Participants}

Participants were recruited as part of an on-going larger study on human kin recognition (https://osf.io/bxy9z/). At the time of the current analysis, 63 pairs of full siblings (83 women: mean age $=24.12$ years, $S D=6.86$ years; 42 men, mean age $=22.90$ years, $S D=7.15$ years) had completed the study. One participant was excluded as data on the genetic relationship with the sibling was missing.

\section{Procedure}


Participants played a one-shot trust game (TG) (Apicella et al., 2010; Berg, Dickhaut, \& McCabe, 1995), as both the trustor (allocating monetary resources to their sibling) and also as the trustee (receiving monetary resources from their sibling). For the purpose of this exploratory study, we only report the data collected during the trustor phase of the game.

In this role, participants received 5 lab coins, which they could share with their sibling. They were informed that 1 lab coin was the equivalent of 50 pence $(£ 0.50, \mathrm{GBP})$ in real monetary terms. Participants were asked to transfer any amount from 0 to 5 lab coins (in 1 lab coin increments) to their sibling by selecting their answer from a drop-down menu. Participants were informed that the number of coins they sent to their sibling would be tripled and that they could keep any remaining coins that they did not send. Subjects were only informed of the amount of lab coins that their sibling transferred to them at the end of the experimental session. Thus, knowledge of their sibling's allocation of coins did not influence their own decision-making process. The amount of lab coins transferred was our measure for trust (Johnson \& Mislin, 2011; Wu et al., 2017). The time spent making the transfer decision was measured in seconds.

\section{Analysis}

Following Evans et al. (2015), decision time was log10 transformed, the amount transferred during the TG was centered and scaled to range from -0.5 to +0.5 , and participant sex was effect coded as -0.5 (women) and +0.5 (men). Linear and quadratic effects of trust on self-paced decision time were estimated in a regression analysis. To avoid multicollinearity, orthogonal instead of raw polynomials were adopted (Shacham \& Brauner, 1997). Analyses were conducted using R v3.6.0 (R Core Team, 2018). Data and analysis code are publicly available at (https://osf.io/82sra/).

\section{Results}

Siblings transferred on average 0.88 of their initial endowment, which was higher than mean reported in previous meta-analysis (average percentage = 0.5 ; Johnson \& Mislin, 2011). No sex differences regarding amount transferred $(\mathrm{t}=-0.48, \mathrm{df}=123, \mathrm{p}=0.635)$ or self-paced decision time $(\mathrm{t}=$ 0.35 , df $=123, p=0.728$ ) were found (Figure 1 ). 

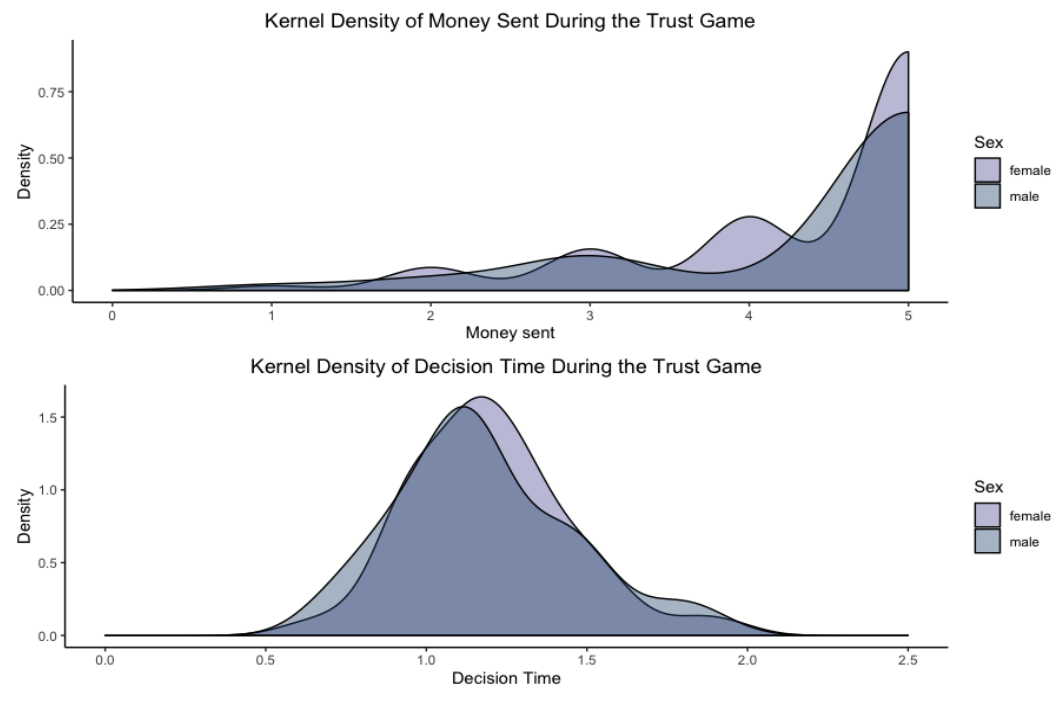

Figure 1: Kernel densities of money sent and decision time during the trust game by sex.

We conducted a linear regression analysis with log-transformed self-paced decision time as a dependent variable and trust scores (centered and scaled to range from -0.5 to +0.5 ) as predictor. Results showed a significant negative effect of trust scores on decision time (estimate $=-0.33, t=-3.76, p<.001$ ) (Figure 2).

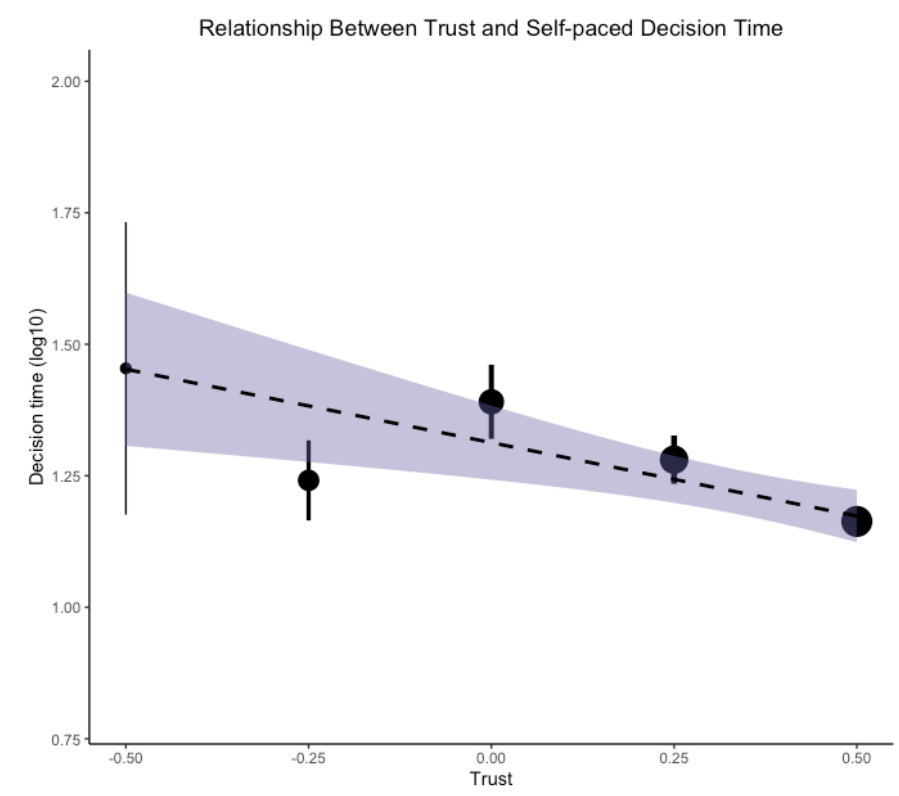

Figure 2: Linear effect of trust on decision time. Money sent was binned into 5 categories and dot sizes are proportional to number of observations in each category. Error bars indicate standard error.

We then tested a quadratic relationship between trust and decision time. Since the linear and quadratic terms were highly correlated $(r=0.77, \mathrm{p}<$ 0.001 ), orthogonal polynomials were adopted in the regression analysis to reduce the collinearity between terms. A significant negative effect of linear 
term (estimate $=-0.93, \mathrm{t}=-3.7875, \mathrm{p}=0.0002$ ), but not the quadratic term (estimate $=-0.39, t=-1.59, p=0.114$ ), was found on decision time.

Following Rand et al. (2012), additional analyses were conducted by excluding participants with decision times more than three standard deviations above the mean and the results remained qualitatively similar to the main analyses.

\section{Discussion}

Results from our study showed that a linear rather than a quadratic effect explains the relationship between trust and self-paced decision time when siblings are interacting in a one-shot trust game. This finding is consistent with past studies showing a negative relationship between cooperation and decision time in non-kin individuals (e.g. Lotito et al., 2013; Rand et al., 2012).

At least in the context of the trust game, siblings seem to apply intuitive strategies when making prosocial decisions. This is also consistent with the hypothesis that an unconscious psychological mechanism regulates prosocial behaviors toward kin specifically (Lieberman et al., 2007). Therefore, these behaviors seem to be processed faster when siblings are making decisions.

However, one alternative explanation for the negative relationship between trust and decision time in our study relates to the number of extreme cooperative decisions observed (i.e. participants who transferred the whole endowment). Evans et al. (2015) argue that the association between decision time and cooperation is drifted by extreme decisions: a negative relationship arises when there are many extreme cooperative decisions, while a positive relationship arises when there are many extreme selfish decisions.

In conclusion, we found a negative linear, rather than quadratic, relationship between trust and self-paced decision time when siblings make transfers during the trust game. This suggests that decisions among siblings appear to be based on intuitive and effortless processes.

\section{Funding}

This research was funded by an ERC grant (KINSHIP) awarded to LMD.

\section{Competing interests}

The authors have no competing interests to declare.

\section{References}

Apicella, CL, Cesarini, D, Johannesson, M, Dawes, CT, Lichtenstein, P, Wallace, B, ... \& Westberg, L (2010). No association between oxytocin receptor (OXTR) gene polymorphisms and experimentally elicited social preferences. PloS one, 5(6), e11153. 
Berg, J, Dickhaut, J, \& McCabe, K (1995). Trust, reciprocity, and social history. Games and economic behavior, 10(1), 122-142.

Cappelen, AW, Nielsen, UH, Tungodden, B, Tyran, JR, \& Wengström, E (2016). Fairness is intuitive. Experimental Economics, 19(4), 727-740.

DeBruine, LM (2002). Facial resemblance enhances trust. Proceedings of the Royal Society of London. Series B: Biological Sciences, 269(1498), 13071312.

Evans, AM, \& Rand, DG (2019). Cooperation and decision time. Current opinion in psychology, 26, 67-71.

Evans, AM, Dillon, KD, \& Rand, DG (2015). Fast but not intuitive, slow but not reflective: Decision conflict drives reaction times in social dilemmas. Journal of Experimental Psychology: General, 144(5), 951.

Halali, E, Bereby-Meyer, Y, \& Meiran, N (2011). When rationality and fairness conflict: The role of cognitive-control in the ultimatum game. Available at SSRN 1868852.

Hamilton, WD (1964). The genetical evolution of social behaviour. II. Journal of theoretical biology, 7(1), 17-52.

Johnson, ND, \& Mislin, AA (2011). Trust games: A meta-analysis. Journal of Economic Psychology, 32(5), 865-889.

Kahneman, D (2011). Thinking, fast and slow. Macmillan.

Lieberman, D, Tooby, J, \& Cosmides, L (2007). The architecture of human kin detection. Nature, 445(7129), 727.

Lohse, J, Goeschl, T, \& Diederich, JH (2017). Giving is a question of time: response times and contributions to an environmental public good. Environmental and resource economics, 67(3), 455-477.

Lotito, G, Migheli, M, \& Ortona, G (2013). Is cooperation instinctive? Evidence from the response times in a public goods game. Journal of Bioeconomics, 15(2), 123-133.

Park, JH, \& Schaller, M (2005). Does attitude similarity serve as a heuristic cue for kinship? Evidence of an implicit cognitive association. Evolution and Human Behavior, 26(2), 158-170.

Piovesan, M, \& Wengström, E (2009). Fast or fair? A study of response times. Economics Letters, 105(2), 193-196.

R Core Team. (2018). R: A language and environment for statistical computing. Vienna, Austria: R Foundation for Statistical Computing. Retrieved from https://www.R-project.org/ 
Rand, DG (2019). Intuition, Deliberation, and Cooperation: Further MetaAnalytic Evidence from 91 Experiments on Pure Cooperation. Available at SSRN 3390018.

Rand, DG, \& Nowak, MA (2013). Human cooperation. Trends in cognitive sciences, 17(8), 413-425.

Rand, DG, Greene, JD, \& Nowak, MA (2012). Spontaneous giving and calculated greed. Nature, 489(7416), 427.

Schlomer, GL, Del Giudice, M, \& Ellis, BJ (2011). Parent-offspring conflict theory: An evolutionary framework for understanding conflict within human families. Psychological review, 118(3), 496.

Shacham, M, \& Brauner, N (1997). Minimizing the effects of collinearity in polynomial regression. Industrial \& engineering chemistry research, 36(10), 4405-4412.

Spiliopoulos, L, \& Ortmann, A (2018). The BCD of response time analysis in experimental economics. Experimental Economics, 21(2), 383-433.

Vollan, B (2011). The difference between kinship and friendship:(Field-) experimental evidence on trust and punishment. The Journal of SocioEconomics, 40(1), 14-25.

Wu, J, Balliet, D, Tybur, JM. Arai, S, Van Lange, PA, \& Yamagishi, T (2017). Life history strategy and human cooperation in economic games. Evolution and Human Behavior, 38(4), 496-505.

Zaki, J, \& Mitchell, JP (2013). Intuitive prosociality. Current Directions in Psychological Science, 22(6), 466-470. 